\title{
CONFIGURACIONES SOBRE EL SENTIDO DEL JUEGO PARAEL PUEBLO ANCESTRAL MISAK DEL RESGUARDO GUAMBIA
}

\section{MEANING CONSTRUCTIONS OF THE GAME FOR THE MISAKANCESTRAL PEOPLE IN COLOMBIA}

\author{
Germán Darío Isaza Gómez', \\ Libardo Córdoba Rentería², \\ Ana María Bedoya Taborda ${ }^{3}$ \\ Institución Universitaria Escuela Nacional \\ del Deporte \\ Grupo de Investigación Educar 2030 - \\ Grupo de Investigación Estudios Aplicados \\ al Deporte IUEND
}

\section{RESUMEN}

Colombia es un país pluricultural, multiétnico, aún la habitan alrededor de 103 Pueblos Indígenas; además, se conservan 65 lenguas propias de las comunidades nativas. El

12 Doctorando en Educación, Universidad de la Salle de Costa Rica, Magister en Educación, de la Universidad de Caldas, docente - Investigador de la Institución Universitaria Escuela Nacional del Deporte. Director del Grupo de Investigación Educar 2030. Correo electrónico german. isaza@endeporte.edu.co https://orcid.org/0000-0001-84759994

2

Magister en Educación Popular. Docente - Investigador Institución Universitaria Escuela Nacional del Deporte. Director del Grupo de Investigación Estudios Aplicados al Deporte. Correo electrónico libardo.cordoba@endeporte.edu.co https://orcid.org/0000-0003-2500-5361

$3{ }^{3}$ Profesional en Deporte, Institución Universitaria Escuela Nacional del Deporte. Semillero de Investigación Educar 2030. Correo electrónico ana.bedoya@endeporte. edu.co https://orcid.org/0000-0003-3722-879X objetivo del estudio fue comprender el sentido del juego para la comunidad Indígena Misak del resguardo la Marquesa, ubicada en el departamento del Cauca. El estudio siguió la lógica de la investigación acción, la cual se orientó desde la lógica introspectivo vivencial, donde se utilizó la observación etnográfica participante y no participante, entrevistas a profundidad como instrumentos importantes para condensar los elementos significativos $y$ recurrentes que suceden alrededor del juego. Comprender los diversos juegos como el Tsarap Lulepik, Chanchiku, Tsik Pala Nepuna Ampik, Lasrúarúk y el Dchama, constituyen una forma cultural de comprender sus luchas por su pervivencia ancestral, sino una conexión con 
su pensamiento de sus mayores desde el Isup; generando una convivencia con la naturaleza, la vida y su territorio a través del Aship; conectando las vivencias de los ancestros, quienes viven a plenitud desde el Merep; las cuales se relacionan con las diversas actividades de la vida cotidiana de la comunidad desde el Marep.

PALABRAS CLAVE: Juego, cultura, cosmogonía, costumbres y tradiciones.

\section{ABSTRACT}

Colombia is a multicultural, multiethnic country, it is still inhabited by about 103 Indigenous Peoples, and 65 native languages are also preserved. The objective of the study was to understand the meaning of the game for the Misak Indigenous community of La Marquesa shelter, located in the department of Cauca. The study followed the logic of action research, which was oriented from experiential introspective logic, where participant and non-participant observation was used, in-depth interviews as important instruments to condense the significant and recurring elements that happen around the game. Understanding the various games such as Tsarap Lulepik, Chanchiku, Tsik Pala Nepuna Ampik, Lasrúarúk and Dchama, constitute a cultural way of understanding their struggles for their ancestral survival, but a connection with their thinking of their elders from the Isup; generating a coexistence with nature, life and its territory through the Aship; connecting the experiences of the ancestors, who live fully from the Merep; which are related to the various activities of the daily life of the community from the Marep.

KEYWORDS: Game, culture, cosmogony, customs and traditions

\section{RESUMO}

A Colômbia é um país multicultural e multiétnico, aindaéhabitada porcerca de 103 povos indígenas e 65 idiomas nativos também são preservados.
O objetivo do estudo foi compreender o significado do jogo para a comunidade indígena Misak do abrigo La Marquesa, localizado no departamento do Cauca. O estudo seguiu a lógica da pesquisa-ação, que foi orientada a partir da lógica introspectiva experiencial, na qual foi utilizada a observação participante e não participante, entrevistas em profundidade como instrumentos importantes para condensar os elementos significativos e recorrentes que ocorrem ao redor do jogo. Compreender os vários jogos como Tsarap Lulepik, Chanchiku, Tsik Pala Nepuna Ampik, Lasrúarúk e Dchama, constitui uma maneira cultural de entender suas lutas pela sobrevivência ancestral, mas uma conexão com o pensamento de seus anciões do Isup; gerando uma convivência com a natureza, a vida e seu território através da nave; conectando as experiências dos antepassados, que vivem plenamente do Merep; que estão relacionados às diversas atividades da vida cotidiana da comunidade do Marep.

PALAVRAS-CHAVE: Jogo, cultura, cosmogonia, costumes e tradições

\section{INTRODUCCIÓN}

En el presente estudio aborda una descripción amplia del sentido del juego para la comunidad indígena Misak del resguardo ancestral Guambía, ubicado en el municipio Silvia, Cauca. Este estudio se abordó desde la Electiva de Ampliación Profesional Deporte y Mediaciones Educativas adscrito al Grupo de Investigación Educar 2030 de la Institución Universitaria Escuela Nacional del Deporte.

Colombia es un país con gran diversidad étnica, es pluricultural, actualmente cuenta con 103 Pueblos Indígenas reconocidos según el reporte de la Organización Indígena de Colombia ONIC- citado en los Lineamientos de Política Pública de Coldeportes (2018); además en la actualidad persisten 65 lenguas de propias de 
los pueblos indígenas, según Corrales (2018) existen diversos orígenes de estas lenguas "65 lenguas indígenas tienen diverso origen; 51 de ellas provienen de 13 familias lingüísticas, mientras que ocho se consideran aisladas. Estas lenguas son habladas por aproximadamente 400.000 personas" (p.3)

Uno de los Pueblos Indígenas que está en permanente lucha por su reconocimiento $y$ participación como grupo étnico es el Pueblo Misak, quienes se denominan hijos del agua, la palabra y los sueños -Piurek-. La cosmogonía Misak considera una cosmovisión dual de la naturaleza, para ellos existe lo femenino y lo masculino; el sol y la luna; lo frio y lo caliente; espíritus benéficos y maléficos. Actualmente tienen una organización de gobierno propia, sistema de salud y forma de educación, en la que se destaca la Universidad Misak. Su lengua es la Nam Trik, la cual pertenece a la familia lingüística Chibcha. Es un pueblo que se aferra y lucha constantemente por su pervivencia, territorio y cultura, Medina \& Cantero (2018) en su libro Interculturalidad en las etnias de Colombia, recuerdan que la corte constitucional de Colombia advirtió "entre 2009 y 2010 al menos 35 grupos indígenas se encontraban en peligro de extinción a causa del conflicto armado y el desplazamiento" (p.11) entre ellos los Misak, a causa de su ubicación geográfica en el Cauca colombiano, uno de los departamentos más golpeados por la violencia, producto del el conflicto armado (Machuca, 2017).

Los Misak nunca han dejado de luchar, poseen gran resiliencia, y se niegan a desaparecer, han luchado desde los tiempos de la colonia por su territorio y cultura. Ahora la globalización, la tecnología, el intercambio cultural han permeado su territorio, y desde luego la globalización del deporte ha llegado a su territorio. Actualmente, se práctica el fútbol, el baloncesto y otras actividades deportivas ajenas a su cultura, generando una tensión permanente entre sus tradiciones ancestrales y los deportes modernos. Está lucha por sus tradiciones, cosmovisión y cosmogonía se conserva en la actualidad con sus múltiples formas y manifestaciones a través del juego, el cual se propone en este estudio como una lucha simbólica para los Misak por conservar sus tradiciones.

En este orden de ideas, es preciso realizar un recorrido por las diferentes concepciones instituidas que se tiene sobre el concepto de juego, Méndez-Giménez \& Pallasá-Manteca (2018); Chiappe, Cardinal, González \& Velásquez, (2019) concuerdan que el juego se define por una dinámica de placer funcional, de tensión al gozo. En la misma línea se sitúa Pugmire- Stoy (1992) quien define el juego como "la participación activa en actividades físicas o mentales placenteras con el fin de conseguir una satisfacción emocional" (p. 20) Entendiéndolo en muchos casos como la pedagogía del disfrute como lo concibe Braudel, (2019).

Tradicionalmente, el concepto del juego se relaciona con el goce y el disfrute, naturalmente los juegos se realizan cuando los diferentes niños, niñas, jóvenes y adultos poseen tiempo libre, de ahí que es muy natural escuchar afirmaciones como: "Quieres jugar a..." "cuando te desocupes ¿podemos jugar?" "El domingo jugamos fútbol”. En el imaginario instituido existen diferentes tipos de juegos: juegos deportivos, juegos predeportivos, juegos rompehielos, juegos de presentación, juegos de campo, juegos de azar, juegos de persecución, juegos de mesa, juegos interactivos, entre otros, y actualmente emergen juegos cada vez más específicos, los cuales son realizados por los diferentes grupos humanos.

Actualmente el juego es asumido en algunos sectores de la sociedad moderna como una pérdida de tiempo, en algunos casos no se utiliza en actividades que permiten una formación de los niños, niñas y jóvenes; desconociendo en él todos los beneficios y posibilidades que emergen a través de las diferentes manifestaciones del 
juego: Es considerado una herramienta potente para la socialización y el aprendizaje de los niños, niñas y jóvenes; estimula la creatividad, ayuda a la resolución de conflictos, promueve el pensamiento crítico y la toma de decisiones, entre otros Quispillo, Marinela, Cahuantico, \& Marisol, (2020); Cerchiaro-Ceballos, BarrasRodríguez, \& Vargas-Romero, (2019) Salazar, (2018); Loyola \& María, (2016); Lacasa, (2011); Meneses \& Monge, (2001).

Como se puede evidenciar el concepto del juego está totalmente asociado con unas tradiciones y culturas, en diferentes lugares el juego representa y configura una forma particular de ver y vivir la vida, promoviendo espacios de construcción social a través de sus representaciones sociales, las cuales están asociadas con el aprovechamiento del tiempo libre, el goce y disfrute de sus habitantes.

La importancia de este trabajo permite ampliar la mirada del sentido y significado del juego, superando las visiones reduccionistas del goce y el disfrute; reconociendo su papel protagónico en los procesos de construcción social, restableciendo en muchos escenarios las relaciones, dinamizan la vida de los pueblos, permiten unas formas instituidas de vivir en sociedad, por ejemplo, en la zona sur de Colombia, algunos de sus habitantes juegan a la chaza, en el eje cafetero se juega al sapo o la rana: en la costa atlántica se juega dominó, y así diferentes regiones asumen un juego tradicional propio o distintivo de la región.

Es necesario rescatar la importancia del juego en la vida de los individuos, ya que, desde las edades iniciales, el niño y la niña aprende a conocer el mundo jugando, de allí que está asociado con el aprendizaje de las personas. En este orden de ideas, el juego siempre ha estado vinculado con la vida de las personas, ya que para jugar no existe un prerrequisito de edad. Se puede jugar en cualquier momento de la vida, (Yuni \& Urbano, 2005) (Allan, Wachholtz,
\& Valdés, 2005)

El juego, en el ser humano facilita la expresión de diferentes sentimientos: de alegría, de diversión, de amor, o expresión de emociones; los cuales se develan producto de las emociones emergentes a la hora de jugar, además es una forma de develar los diferentes comportamientos que se establecen en las diferentes personas, de la expresión famosa de "En la mesa y en el juego se conoce al caballero y a la dama". En fin, el juego se convierte en una poderosa herramienta para comprender una serie de relaciones culturales que se dan en los diferentes pueblos y comunidades.

En consideración a todas estas cuestiones, la forma de abordar el problema no es desde la lógica deductiva, en tanto que no es de interés del presente estudio indagar por los modos instituidos, estas categorías no interesan encontrarlas en la teoría formal; sino más bien, el presente estudio prioriza la realidad misma que se configura desde el actuar mismo de la cultura Misak, siendo una lógica introspectiva vivencial, la estrategia desde la cual se revela la emergencia y configuración de las categorías de estudio del fenómeno y del problema en cuestión y desde las que se pretender comprender la realidad objeto de estudio. En palabras de Shotter (2001) "Las realidades se configuran en el bullicio de lo cotidiano" (p.19); en una perspectiva desde la vivencia, o sea que la realidad se construye desde ella misma, por ello hay que vivir la realidad para poder entenderla, ello nos permite obtener una comprensión más amplia de la realidad que se está estudiando.

Así las cosas, la lógica desde la cual se abordó el presente estudio es desde el enfoque Introspectivo vivencial, permitiendo un encuentro directo con la realidad misma, asumiendo el problema desde los elementos constituyentes de la realidad social del pueblo indígena Misak, los cuales no están presentes de forma objetiva a la simple vista de los sujetos, 
Se pretende en el siguiente estudio develar el sentido y significado del juego para la comunidad indígena Misak.

\section{MÉTODO}

El enfoque de investigación se basa en el paradigma cualitativo, según Sampieri, Collado, Lucio, Valencia \& Torres (1998) el cual busca "comprender a profundidad los fenómenos, explorándolos desde la perspectiva de los participantes en un ambiente natural y en relación con el contexto" (p.364), es así como el diseño de este estudio se configuró desde el diseño investigación acción, el cual se abordó desde la propuesta introspectivo-vivencial desarrollada por Padrón (1998):

Más que definición de una realidad externa, el conocimiento es interpretación de una realidad tal como ella aparece en el interior de los espacios de conciencia subjetiva (De ahí el calificativo de introspectivo). Lejos de ser descubrimiento o invención, en este enfoque el conocimiento es un acto de comprensión (p.34)

Es la hermenéutica la pieza clave en el proceso de comprensión de los sentidos y significados de los escenarios sociales y la función ontológica del juego, en la que intervienen tanto los sujetos como los procesos que devienen a su alrededor, considerando la influencia de las tradiciones, los sujetos, el contexto en la percepción del mundo que se configura en la cultura Misak, desde lo anterior, reconoce los aportes de Husserl (1997), en cuanto que la realidad se configura en los sujetos, en este caso desde las formas de participación en las construcciones instituidas que devienen a través de juego en el Pueblo Misak; pues son ellos quienes asumen los elementos de una cultura, dándole sentido a cada uno de ellos y proyectando su vida según ese sentido dado y filosofía ancestral.

El resguardo ancestral de Guambia está ubicado en las estribaciones de la cordillera central al nororiente del municipio de Silvia Cauca. Según Cabildo (2008), el resguardo de Guambia en sus dinámicas de reagrupamiento como grupo étnico, se constituye en 4.582 familias y 22.987 personas, de los cuales 25 familias son Nasas con 273 habitantes. En el resguardo colonial vive el $64.27 \%$ de la población Misak y el $0.54 \%$ de las familias Nasas, para un total en el resguardo ancestral de $64.87 \%$. En los últimos veinte años ha habido una migración del 35 . 06\%, representado en el $30.7 \%$ de las familias que tienen organización de Cabildo y resguardos constituidos, el $3.38 \%$ viven en resguardos de otras comunidades y el $0.98 \%$ viven en las ciudades.

Las técnicas e instrumentos utilizados para recolectar la información fueron la observación participante y no participante, donde los datos relevantes y recurrentes se condensaron en el instrumento diario de campo, los cuales permitieron comprender la realidad sustantiva que emerge a través del juego, comprendiendo sus costumbres, ideologías, cosmovisión y cosmogonía al interior de la cultura Misak. Las entrevistas se planificaron de tal forma que se intentó conseguir los testimonios de un grupo de personas con gran reconocimiento dentro del Cabildo donde se encontraban: Estudiantes de la Universidad Misak, un integrante de la guardia Indígena, un líder del cabildo de jóvenes estudiante de la Universidad del Valle, un Sanador, dos Mayores y dos mujeres adultas del resguardo.

El diseño de la investigación permitió abordar el estudio en tres momentos de análisis: En el primer momento se realizó el proceso de acercamiento a la realidad, a partir de la información contenida en el diario de campo, se asumió un proceso de codificación por medio de una categorización abierta, para ello se empezó a identificar los aspectos relevantes y recurrentes dentro del Pueblo Misak relacionado con el desarrollo de los juegos y los relatos de 
los Mayores, los cuales fueron la base inicial de acercamiento con el objeto de estudio bajo un análisis de significatividad; para Ritzer (1996) "Lo que es visible es una realidad que esconde a otra, una realidad más profunda que se oculta, y el descubrimiento de esa realidad, construye el verdadero propósito de la investigación social y humana" (p. 45). Este acercamiento gradual a la realidad permitió inicialmente ir configurando $\mathrm{y}$ redescubriendo la realidad del sentido $\mathrm{y}$ significado del juego en el Pueblo Misak.

El segundo momento, se dio a partir de la codificación axial, allí se seleccionaron a los informantes, quienes fueron elegidos de acuerdo con la percepción de los investigadores frente a las posibilidades de configuración y reconfiguración de los juegos significativos para el Pueblo Misak, se depuraron las categorías de estudio por medio de la teoría sustantiva y la teoría formal.

En el tercer momento, de profundización durante la codificación selectiva, se logró develar los juegos más significativos de los habitantes del Pueblo Indígena Misak, configurando el sentido y significado a estas prácticas ancestrales. Además, la entrevista semiestructurada, fue un instrumento clave para comprender la lucha misma de los juegos y su papel protagónico en la vida instituida y ancestral dentro de la cultura Misak, los cuales han logrado pervivir generación tras generación, en una lucha constante por no desaparecer producto de la influencia de la globalización sobre las nuevas generaciones de jóvenes que habitan el Cabildo.

Para el desarrollo de la investigación se logró el consentimiento del Taita del resguardo, el cual se logró a través de la armonización y limpieza de los investigadores a través de una serie de rituales propios de la cultura Misak, los cuales fueron prerrequisitos indispensables antes de iniciar el proceso de recolección de la información, a saber, el proceso de armonización tuvo las siguientes experiencias:
Armonización corporal a través de semillas de árboles, con los cuales se realizó una especie de instrumento musical de viento, con el cual se recorrió el cuerpo de los investigadores, con el fin de limpiar el cuerpo o Pishimaruk y ponerlo en sintonía con la Pachamama. El Rapé: El cual consistió en insertar un tubo pequeño por la nariz y se le inyecta un polvo, el cual es soplado por el médico chamán ${ }^{4}$ llamado Murbik.

Luego de este proceso se pudo conversar con el Taita $^{5}$ de la comunidad, quien además ofreció a los investigadores Ayaguasca, infusión de hojas de plantas parecido al Yahé, el cual se utilizó para despertar los sentidos, igualmente se realizó una ceremonia de punción que consistió en ubicar unas agujas en cada brazo, con el fin de perder los miedos, las inseguridades propias que se presentaran durante el proceso. Este fue el proceso inicial de purificación y armonización el cual dio apertura al proceso de recolección de la información.

En este sentido el proceso de recolección de la información estuvo acompañado de un proceso de purificación permanente, el cual consistió en realizar otras dos actividades propias del Pueblo Misak, una consistió en la participación en una actividad llama el Palabreo, el cual consistió en una actividad donde se purifica la palabra en la Universidad Misak, donde era indispensable mambear hoja de coca durante la actividad, la cual tuvo una duración de 24 horas, donde se purifica la palabra y se obtiene la sabiduría para entender los que se dice, y otra actividad de purificación del cuerpo, a través de una limpieza corporal en una cascada ubicada en el macizo colombiano, en el páramo de las Delicias, la cual consistió en entrar al agua y aguantar el mayor tiempo posible el agua fría que corría allí, los

\footnotetext{
$4 \quad$ Del fr. chaman, y este del tungús šaman. $m$. y $f$. Persona a la que se supone dotada de poderes sobrenaturales para sanar a los enfermos, adivinar, invocar a los espíritus.

$5 \quad m$. infant. rur. Bol., Chile, Col., Cuba, Ec., Hond. y R. Dom. U. para dirigirse o aludir al padre y a las personas que merecen respeto. U. t. c. coloq. Taita persona con poder político dentro de la comunidad.
} 
Misak tienen una conexión vital con el agua, ya que para ellos es sagrada, es la vida misma.

El análisis de la información se realizó a partir de la codificación de los diarios de campo y las entrevistas en el software de investigación cualitativa NVivo, logrando configurar todo un entramado de sentido y significaciones a partir de la realidad instituida que deviene en la comunidad Misak del resguardo la Marquesa.

\section{RESULTADOS}

Para una mejor comprensión de los resultados, inicialmente se describen los juegos propios que se realizan en el Pueblo Misak del resguardo Guambía; luego, se presenta el sentido del juego para los indígenas Misak a partir de su cosmovisión.

Los juegos propios del Pueblo Indígena Misak son: Tsarap Lulepik, Chanchiku, TsikPala Nepuna Ampik, lasrúarúk y Dchama, para una mejor comprensión de estas actividades, primero, se realiza una descripción de los elementos con los que se debe jugar (materiales), luego, el objetivo y finalmente, una descripción del cómo se juega.

\section{Tsarap Lulepik o Zarambico}

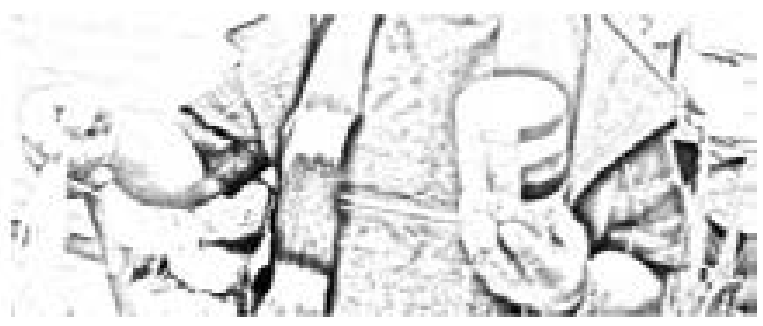

Imagen 1..Tsarap Lulepik o Zarambico.

Para poder jugar este juego, se necesita un zarambico o trompo adaptado, un palo de madera pequeño al que se le clava una guasca y varias cabuyas. El zarambico y la guasca deben ser elaboradas de madera de cerote de páramo o de encenillo.
El objetivo del juego consiste en que el jugador debe hacer girar el trompo lo que más pueda con un látigo, controlar el movimiento para que el zarambico no se choque contra la pared.

Se juega enrollando la guasca en el zarambico, se acuesta en el suelo, y con un movimiento rápido y ágil el jugador debe desenrollar el zarambico (trompo) de la cuerda para que pueda girar, al zarambico hay que darle "juete" con la guasca para que pueda girar el mayor tiempo posible.

Este juego se práctica en grupos o individual, en grupos se delegan funciones donde uno hace girar el trompo y los otros 4 le pegan con el látigo al trompo, logrando que no deje de girar, también lo juegan en zonas diferentes como por ejemplo en canchas o zonas grandes en donde deben lleva el trompo de un lado al otro sin que el trompo deje de bailar.

\section{Chanchiku o Carreto}

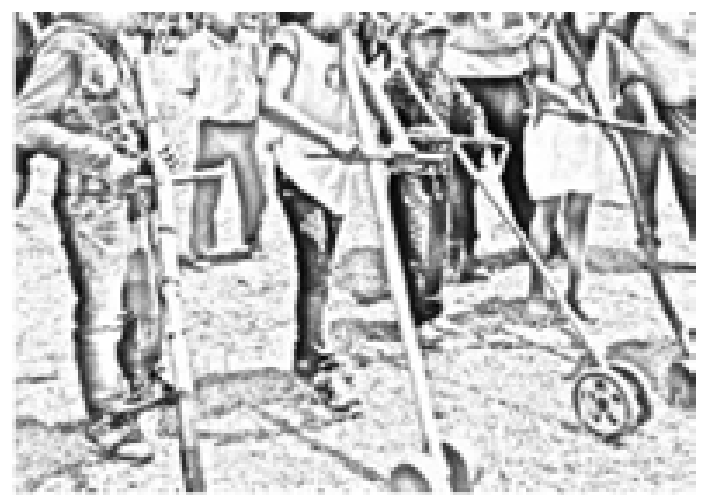

\section{Imagen 2. Chanchiku o Carreto}

Para poder jugar este juego se necesita madera de motilón o de cerote del páramo y dos ruedas. Al palo de madera se le atraviesa un palo de madera en el tercio superior, en la parte inferior se le atraviesa uno más pequeño en el cual van las dos ruedas.

El objetivo del juego consiste en ayudar a transportar objetos pesados, agua o alimentos a su hogar de un punto a otro. 
Este juego se práctica en la vida cotidiana de los indígenas Misak ya que con ella se transportan diversos elementos a sus hogares o de sus hogares a otros lugares.

\section{Tsik Pala Nepuna Ampik o Zancos}

Para poder jugar este juego se necesitan los Zancos hechos de palos de madera y cabuya, sobre ellos debe subirse la persona para lograr desplazarse.

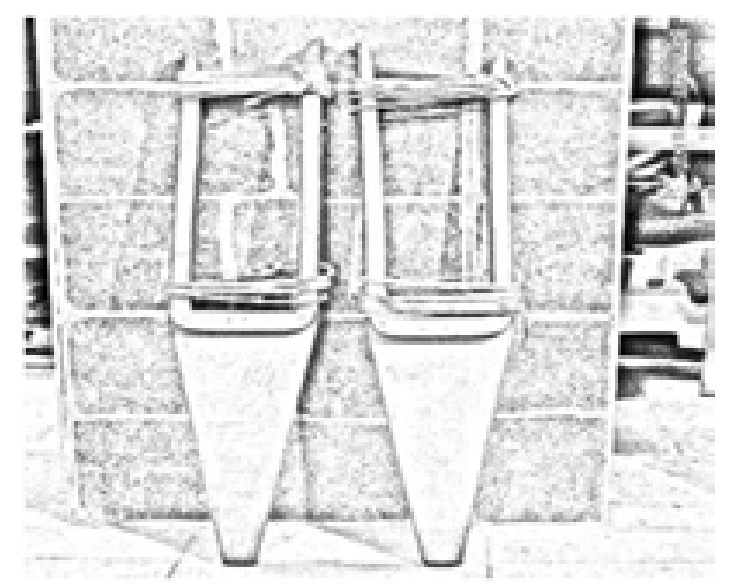

Imagen 3. Tsik Pala Nepuna Ampik o Zancos

El objetivo del juego es caminar el mayor tiempo posible en unos palos de madera sin caerse.

Se juega, subiendo los pies sobre los palos de madera, con las manos se debe agarrar la parte de arriba de los zancos. Para evitar caerse de los Zancos, no pueden quedarse quietos en un solo punto, sino que todo el tiempo deben estar en dando los pasos o zancadas. Una vez se dominan los Zancos, se pueden realizar competencias sobre ellos. Una de estas competencias consiste en que los participantes atraviesen un terreno sin caer, se establece un punto de salida y de llegada, el participante que pueda a travesar la pista, el terreno o el barro sin caer es el ganador. La persona que más dure caminando con ellos, sin caer, también es ganador.

\section{Lasrúarúk o Arco y flecha}

Para poder jugar este juego se necesita arco, flechas y tablero.

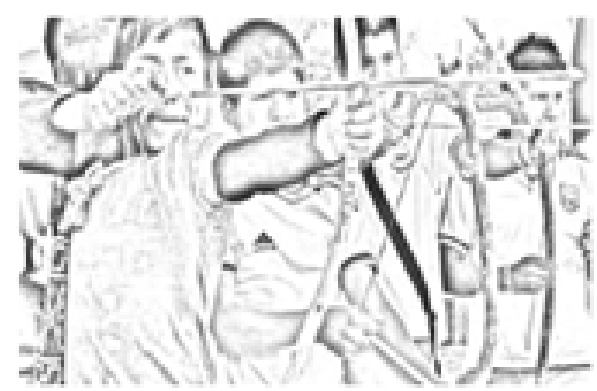

Imagen 4. Lasrúarúk o Arco y flecha

El objetivo del juego consiste en lanzar una flecha con un arco y ubicarlo en el centro del tablero o sobre un punto determinado.

Se juega delimitado con círculos la puntuación $100,80,60,40,20$ de acuerdo donde caiga la flecha, el jugador tendrá tres oportunidades para lanzar la flecha, al finalizar gana quien obtenga mayor puntaje. El juego aplica además ubicando unos puntos de referencia en árboles frutales, quien logre derribar el fruto seleccionado también es ganador.

\section{Dchama o Zumbambico}

Para poder jugar este juego se necesita madera y cabuya.

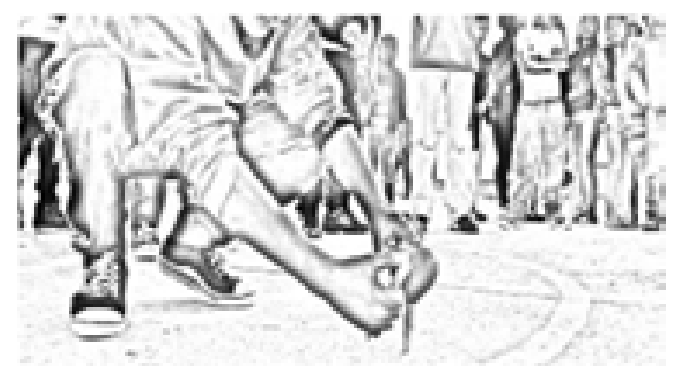

Imagen 5. Dchama o Zumbambico

El objetivo del juego consiste en poner a girar el zumbambico más tiempo que los demás participantes. 
Para jugarlo el jugador deberá enrollar el zumbambico con una piola y soltarlo con fuerza para que este gire.

\section{SENTIDO DEL JUEGO A TRAVÉS DE LA COSMOVISIÓN MISAK}

En el segundo momento de los resultados se presenta el sentido los juegos para el Pueblo Misak, implicó una introspección en la realidad propia de la cosmogonía, conocer sus ritos, costumbres y formas de organización. Para realizar este proceso de indagación fueron necesarias ceremonias de limpieza y equilibrio energético, donde se armonizó la vida de quienes realizaron el proceso de recolección y análisis de la información, favoreciendo una conexión con la madre tierra; además se participó en clases propias del Pueblo Misak para identificar algunas nombres que se les da en su idioma a las montañas y lagos que componen su territorio; se obtuvo conocimiento de los tejidos y su significado en morrales, sombreros y formas de vestir; además, se pudo evidenciar formas de intercambio como el trueque, los procesos propios de educación en su territorio, inclusive se pudo asistir a la Universidad Misak y participar de un encuentro académico que fortaleció el intercambio de saberes; permitiendo una vivencia de sus saberes más íntimos, donde se tuvo la posibilidad de interactuar con el yahé, el rapé, el palabreo.

En este orden de ideas, abordar el sentido, significa una construcción imaginaria de la cultura Misak, cargada de símbolos y representaciones de la vida ancestral, para ello, se realizó una correlación de estos juegos con los cuatro elementos de la cosmovisión Misak, intentando articular cada juego con el Isup (pensar), Aship (ver), Merep (sentir) y el Marep (hacer); permitiendo comprender la riqueza de la cosmogonía de los pueblos con sus diversas formas de comprender y vivir la realidad a través su ancestralidad.
La lucha constante del pueblo Misak por conservar su cultura, ancestralidad y cosmovisión es una tarea compleja, producto de la globalización, la occidentalización del pueblo ha generado una tensión por la pervivencia de sus tradiciones. El pueblo Misak están sufriendo una tensión con el Misak - Misak en todos los espacios-tiempos finitos e infinitos del Nupirau - Macrocosmos, lo que ha conllevado al desequilibrio energético de los Misak con todos sus congéneres y ciclos de las aguas, tierras, astros, semillas, alimentos medicinas en todos los ciclos de vida Misak, por ello reconstruir el sentido del juego y su aporte a la cosmovisión Misak es una de los aportes significativos del estudio, con el fin de comprender la realidad de la riqueza ancestral que existe a través de su cosmogonía las cuales se pueden evidenciar a través del juego.

\section{Isup: (pensar)}

Esta práctica propia de los Misak, viene del pensamiento ancestral, de los Taitas o Mayores, el zarambico es una práctica que también se desenrolla en grupos familiares o equipos de amigos donde existe una coordinación de ideas para llegar de un punto a otro. los Zancos, aunque es una práctica individual su proceso de creación se desarrolla en equipo, en diferentes espacios con la familia, amigos y compañeros afianzan los lasos familiares. Dentro del pensamiento Misak la práctica del arco y la flecha siempre ha sido un desarrollo de supervivencia y respeto por las tierras.

El Isup para los Misak se refleja en el pensamiento ancestral, y su conexión con el Kaampáwam, el cual es la columna vertebral que da origen a la sabiduría y conocimiento de la vida ancestral Misak - Misak ${ }^{6}$ quienes a través del juego comparten la sabiduría en los diferentes procesos jugados, desde la elaboración de los materiales artesanales para $6 \quad$ Se encuentra presente en los elementos elementales del macrocosmos como la gente agua y gente humana Misak. 
los diferentes juegos; los cuales armonizan las relaciones al interior de las familias y el pueblo, promoviendo una armonía con la naturaleza y los quehaceres de la vida cotidiana, además se realizan para el cuidado de todo el pueblo y en defensa propia. El pensamiento Misak busca el beneficio colectivo de la familia, y la autoridad en la comunidad, donde se prioriza el bien general Nupirau o macrocosmos donde se habla de la existencia de un ir y venir de los espacios-tiempos infinitos y finitos que se mueve constantemente en comunicación y diálogo entre los seres humanos y la naturaleza.

\section{Aship: (ver)}

Los Indígenas Misak son co-creadores de cada juego fomentando su uso en la vida cotidiana ya sea para complementar en su entorno familiar, o para la con-vivencia con sus hermanos en el resguardo, el zarambico es una forma de juego particular donde se conecta la vida misma, "el Zarambico a medida que va girando es la vida de-mostrando cómo son los espirales de cada camino" (DC03 RL08), estos configuran la movilidad del mundo, de la vida, permitiendo un conocimiento de la naturaleza, la vida, la cultura y el territorio a través del juego.

El Carreto ha sido una actividad que se ha desarrollado por muchos ciclos de vida de los Misak donde sus Mayores y Taitas lo utilizaban como una alternativa para poder lograr que los niños y niñas asumieran responsabilidades de su resguardo, sin considerar esta colaboración solo como una obligación de cargas, por el contrario, era la forma de alivianar cargas y equilibrar la fuerza en los niños, fomentando disciplina y trabajo colaborativo al interior de la familia y el resguardo.

Este juego se realizó por muchos años como una alternativa de caza, los grandes Taitas nombraban que ellos predecían este juego donde al final se convertiría en una herramienta de protección para el Pueblo Indígena Misak ante un estado globalizado.

Está practica se desarrolla desde el punto de equilibrio de cada Misak es la observación del movimiento con sentido al lugar que estamos ubicados espacio tierra y tiempo como lo afirma un joven apasionado por los juegos propios Misak "recuerdo el Zumbambico como un juego que tiene la forma del universo, al jugar de esa forma mirábamos como el universo giraba y la tierra como estaba equilibrada y cuando los cultivos estaban o no estaban listos para sembrar, mirábamos qué productos podríamos sembrar y qué productos no."

\section{Merep: (sentir)}

Son tiempos inmemorables, que llevan inmersos los espacios-tiempos infinitos y sus interrelaciones dimensionales por medio del srem, es la velocidad y distancia instantánea con que se mueven los microcosmos para el orden del macrocosmos. Son los ancestros humanos misak, quienes vivenciaban a plenitud el saber y estar siendo misak-misak en todo espaciotiempo en obedecian al kampáwam como principio de orden y salud integral misak-misak en su concepción del Nupirau o macrocosmos.

El cuerpo, la naturaleza y el cosmos para los Misak debe estar en un total equilibrio como lo afirma uno de los Taitas "uno desarrolla las tres energías, el fuego, el agua y el viento. Primero aprender a pensar a través del fuego -el fogón como tal-, el espíritu del agua, a través de la fuerza me invita a coger fuerza en mi cuerpo, pero también el viento, que es el que me ayuda a ser frágil" en cada juego se constituyen estas energías fundamentales en el cuerpo y espíritu de cada Misak.

Practicas del zarambico han sido desarrolladas bajo una percepción de los sentidos completamente equilibrada para poder mantener el trompo en un movimiento constante 
evitando que este choque alrededor de una superficie; está asociado a la ritualidad de la vida, el equilibrio del cuerpo y los órganos de los sentidos, favoreciendo un equilibrio constante a nivel corporal y espiritual.

El juego de los zancos ha sido considerado desde el sentir como una exploración del cuerpo a través del movimiento, como lo afirma un habitante de la comunidad. Esta práctica la conservan los grandes maestros donde se revela un choque de sentires frente a lo implica la realización de este ejercicio como defensa propia de las autoridades, los médicos chamanes y los taitas ven este juego como un propósito de caza y entretenimiento. La construcción de estos utensilios permite la unión familiar, para ellos son sagrados en defensa de la familia y el territorio, allí toda la comunidad está involucrada con la práctica de este oficio, la cual permite la pervivencia y caza de diferentes animales. Cada flecha lanzada está mediada por diversos ritos y tradiciones al interior de la cosmogonía Misak.

El zumbambico está asociado a lo mágico, a la armonización y potenciación de las ideas y pensamiento Misak, a esa ritualidad los ancestros hablan y muestran a través del cuerpo, con el uso de los sentidos, es el equilibrio perfecto entre lo físico y lo mental.

\section{Marep: (hacer)}

El juego está totalmente ligado al hacer Misak, se relaciona con las diversas actividades cotidianas de la comunidad, transportar el agua, la leña, los alimentos y los diversos elementos del hacer en la comunidad, algunos juegos son utilizados para alivianar las cargas, armonizar las energías y aportar en la comunidad de forma responsable, conectando sus pensamientos, sentires y creencias a través del juego en una lucha por la pervivencia de sus costumbres y tradiciones.
La elaboración de los Zancos es creación propia de la zona donde están ubicados cada quien se encarga de diseñar y hacer este juego, como lo afirma una de las autoridades indígenas "Los Zancos generalmente los elaboramos cuando uno va a trabajar lo que llamamos "va a tumbar montaña" y uno se encuentra con ciertos palos y los va clasificando entonces generamos una identidad de que estuvo en el "trabajadero" y encontré esos palos y empezamos a jugar, en el mismo lugar de trabajo uno iba mirando con qué cosas iba a jugar, por ejemplo: las plantas que crecian que parecian muñequitos las cortábamos, entonces, es ahí donde empieza la interacción con todo entonces uno como niño aprende a identificar que los juguetes están ahí en nuestro entorno simplemente que hay que cortarlos. La naturaleza de los juegos habita la vida misma de la comunidad en todas sus etapas.

El tiro al arco se convirtió en una práctica solo para las autoridades indígenas, donde la realización de este es con el fin de defender sus territorios y sus tierras, es una práctica que ha sido desarrollada poco a poco por los jóvenes que hacen parte de la autoridad indígena, como lo afirma un joven de la autoridad indígena Misak "anteriormente se utilizaba era para cazar pero dentro de nuestra comunidad hay un choque con esta práctica, la guardia Misak es muy diferente a la guardia indígena, esta practicaba cambiado incluso en su manera de pensar, hay mayores a los que no les gusta, les parece que afecta a la comunidad y al mismo pensamiento o cosmovisión Misak pero, en cierta parte resulta necesario por ser un medio de defensa ante el estado como ha sucedido en estos tiempos" Para poder lanzar la flecha, se debe conectar el espíritu con la armonía de la tierra, de la naturaleza, es necesario poder pensar, sentir y ser uno con la naturaleza y pervivencia de la cosmovisión Misak. Una mujer de la comunidad afirma: "el Carreto es una madera hecha por nosotros mismos, está ligado entre lo que es el 
juego, el quehacer y la vida cotidiana entonces por ejemplo en relación al Carrete va como dos llantas o ruedas y esas ruedas van ahí atravesadas con un palo largo es el que hace que ruede y eso lo utilizábamos para cargar agua incluso es un juego de fuerza, equilibrio y también, es una manera de no sentir tanto el peso"
Comprender el sentido y significado de los juegos para el Pueblo Indígena Misak desborda la realidad tal cual como se conoce en el mundo occidental, ya que el juego hace parte de su cultura y tradición. A continuación, en el gráfico 1 , se presentan los diversos elementos constitutivos en donde el juego co-ayuda a la pervivencia de la cultura de la cosmogonía para el Pueblo Indígena Misak.

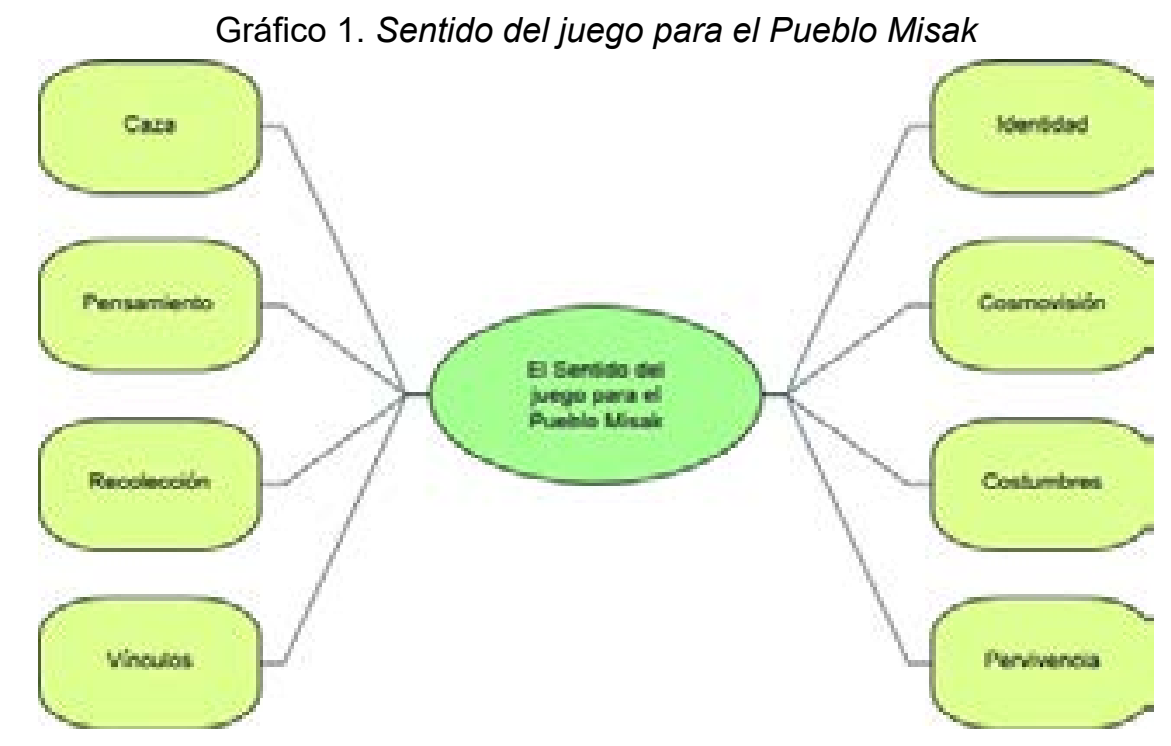

Gráfica 1. Categorías resultantes del sentido del juego para el Pueblo Misak

De acuerdo con el anterior gráfico, se puede comprender que los juegos son una forma de conectar el ser con el entorno, lograr un vínculo sagrado entre la tierra y el ser humano; los juegos hacen parte de los Misak como algo que está ahí en su vida diaria, desde el Isup, Aship, Merep y Marep, conservando la cosmovisión y tradición del resguardo, sin embargo, algunos Taitas aun guardan juegos que no han querido soltar para ser reconocidos por sus nietos, hijos y niños de la comunidad.

\section{DISCUSIÓN}

Intentar comprender el sentido del juego para las comunidades ancestrales implica romper las construcciones instituidas de occidente que se tienen sobre el juego, la cual en muchos casos son construcciones limitadas de la misma realidad, Huizinga (1972) lo define como:

una acción y ocupación libre, que se desarrolla dentro de los límites temporales y espaciales determinados, según reglas absolutamente obligatorias, aunque libremente aceptadas, acción que tiene su fin en sí misma y va acompañada de un sentimiento de tensión y alegría y de ser de otro modo que la vida corriente (p.8).

Las construcciones que actualmente se tienen sobre el juego, se objetivan hacia el goce y el disfrute, donde generalmente está destinada esta acción en las edades iniciales, para Del Toro (2012) "El juego es una actividad placentera, fuente de gozo, además añade que el juego 
es una finalidad sin fin, sus motivaciones son intrínsecas" (p.309)

Para las comunidades ancestrales el juego va más allá de una acción de ocupación libre, mediada por un sentimiento de tensión entre el ganar y el perder; no se realiza una actividad durante el tiempo libre que genere placer, goce y disfrute, estas acciones no son aisladas ni fragmentadas como sucede en la cultura occidental, donde las personas ocupan su tiempo entre la familia, el trabajo, el estudio y, el tiempo que queda (tiempo libre) en muchas ocasiones se utiliza en actividades de ocio, las cuales generan goce y disfrute.

En este orden de ideas en la cultura occidental producto de la globalización, en el afán por el tener, las personas cada vez tienen menos posibilidades de realizar juegos, de compartir con sus seres queridos a través de las diferentes actividades que generen ocio y disfrute, generando cada vez menos tiempo de ocio, donde este tiempo ha sido destinado tradicionalmente a los niños y niñas, ya que a medida que se crece en edad y responsabilidades queda menos tiempo para jugar.

En las comunidades ancestrales es diferente, allí la vida se conecta con el juego, está ligado a las actividades básicas cotidianas de los pueblos indígenas, hace parte de sus interrelaciones ancestrales y culturales de su cosmogonía, y es precisamente desde esta mirada donde los autores pretenden generar una visión más amplia del juego y su impacto en la cultura Misak.

Abordar el sentido del juego desde la cosmovisión Misak es comprender las múltiples interrelaciones ancestrales de su Nupirau y su conexión con el Kaampáwam, entender su relación con la naturaleza, con la comunidad, con la familia, con el cosmos desde el pensar, ver, sentir y hacer propios de un pueblo que lucha por conservar sus tradiciones, ritos y cultura. En este orden de ideas Muñoz \& Yalanda (2017) manifiestan: "los juegos propios en la cultura Misak son importantes porque son la forma en que los niños y niñas aprenden las labores del hogar y las costumbres de la comunidad" (p.64)

Al interior de la comunidad, los Taitas y Mayores nos comparten parte de su pensamiento ancestral y su concepción del juego a través de los tiempos, el cual está anclado a sus raíces desde el nacimiento hasta el final de la vida Misak - Misak, se conservan en su vida cotidiana, no solo en el ser/hacer, sino en el sentir/representar propio de la cosmogonía de la comunidad, donde se pueden oír las voces de sus a través de los relatos:

Yo inicié a los cuatro años con un tizón jugando a las Piramidales, entonces desde ahí uno comienza a comprender la encrucijada y seguir los pasos de los sahumadores, sin embargo, siempre tenemos unos maestros espirituales, desde ahí los maestros nos hablan a través de las señas y los sueños, entonces, a los 9 años ya comencé a interpretar las señas y los sueños a partir de ahí comienza uno a relacionar la vida cotidiana con los juegos propios y es así como nosotros empezamos en juego en mi vida" (DC04RL06).

Comprender los escenarios donde suceden los juegos desbordan la realidad tal cual como se conoce en occidente, quizás puede ser una cultura adelantada en el tiempo, en su forma de concebir la realidad jugada, inclusive se juega durante los sueños, se juega no solo en la vida cotidiana, sino que se considera el juego desde otros "estados espirituales", lo cual desborda la lógica de occidente: analítica y reduccionista de la realidad; demostrando que el juego es muy importante en su vida espiritual. "Jugábamos cuatro niños un mayor y un espíritu" (DC04RL07) 
es muy evidente que en el juego no se trata solo del goce y el disfrute, es un espacio que conecta las realidades de los espacios-tiempos finitos e infinitos del Nupirau o Macrocosmos. Es evidente la relación del juego con los sueños, uno de los Mayores de la cultura Misak afirma:

Los juegos tienen vida, los juegos hablan, el juego tiene conocimiento, el mayor consejero son los sueños desde ahí miro que el juego no era sólo un juego, sino que también tiene algo amplio entre la convivencia y la conciencia (DCO2RLO) ${ }^{7}$.

En el sentido amplio del Nupirau y su conexión con el Kaampáwam, los Misak se han identificado con la naturaleza y el profundo respeto hacia ella, considerando algunas habilidades de los animales en su hábitat como propias, donde se destaca: la agudeza visual, la agilidad, la fuerza, el equilibrio, la velocidad y diversas capacidades físicas las cuales las asumen y representan a través de su cuerpo, a saber uno de los Taitas de la comunidad afirma: "Lo que pasa es que nosotros nos consideramos unos animales simbólicos y a partir de la actividad del juego simbolizamos cualquier circunstancia de la vida" (DC05RL13), en este sentido Toledo (2017) afirma: "actividades corporales, con características lúdicas, y que en la práctica transmitían los mitos, los valores culturales y por tanto congregan en sí mismos el mundo material e inmaterial de cada etnia" (p.1).

El juego además es una representación de la defensa propia, es una lucha constante por la pervivencia de nuestra Kaampáwam, donde cada día el Nupirau y el macrocosmos es afectado por la cultura occidental, basado en leyes, ligada a la religión y el capitalismo, donde el pueblo cada día debe luchar por conservar sus tradiciones, allí el arco, la flecha, la cerbatana han sido

\footnotetext{
$7 \quad$ DC= Diario de Campo 02 corresponde al número
} del relato en el análisis de los datos (RLO= Relato) elementos que no solo ayudan a conservar las tradiciones, son elementos importantes que emergen en la defensa de nuestro Kaampáwam y la forma de pervivir en el tiempo.

Ahora por la tecnología y por toda esta influencia incluyendo la televisión hay niños jugando, pero a pistolas, jugando fútbol, o jugando otra cosa aparte de lo tradicional y lo propio, aunque hay algunas familias que no se ha visto tan permeadas por la cultura de afuera entonces juegan con los mismos elementos que tienen en su medio, sin embargo, la influencia de afuera es cada vez es más fuerte y todo va cambiando (DCO5RL16).

Los juegos han ido evolucionando no solo en occidente, igual sucede en el pueblo Misak, sin embargo, existe una lucha permanente por la identidad de sus juegos propios, tradicionales y autóctonos, donde los Taitas y Mayores generación tras generación intentas trasmitir toda su sabiduría ancestral inclusive a través de los juegos.

Cabe resaltar que se le ha dado un manejo positivo a los juegos de la cultura occidental que a veces han tenido que implementar de manera constructiva para lograr la participación de jóvenes en esta nuevo ciclo de vida donde a través de ellos logran reunirlos y captar su atención para el desenvolvimiento de los temas a tratar en algún encuentro cultural y además comprender que los jóvenes del cabildo han surgido en un pensamiento diferente al de los mayores y sus abuelos taitas por eso en una entrevista con una docente de la escuela mamá manuela afirmaba que: "el pensamiento está ligado a la escuela, los espacios lúdicos, la manera de como también nuevamente se empieza a pensar o repensar la revitalización de los juegos tradicionales la resignificación inclusive va hasta la legitimación misma de todos los saberes ancestrales" (DC02RLO4) 
El juego tiene una puerta con la educación propia, la formación, la reivindicación y el fortalecimiento de la cultura, tiene una puerta grande porque hace un recorrido en el tiempo donde la memoria ancestral juega un papel importante en la enseñanza del Misak desde el juego hasta la educación superior, la vida de cada uno ya inicia no solo como individuo si no como persona, como indígena, como alguien dentro de una comunidad basada en leyes que van desde lo legal hasta lo espiritual, así el juego se convierte en la herramienta para ellos donde involucran el todo por el bien de todos .

El zepcuagoscua, turmequé o tejo, en los territorios chibchas de Colombia, el chaaj o juego de pelota mesoamericano en los territorios mayas de Guatemala estudiados por PanquebaCifuentes, (2012), son expresiones deportivas y recreativas que se niegan a desaparecer en los territorios ancestrales, los juegos que aquí se presentaron son esenciales para la pervivencia de uno de los pueblos indígenas de Colombia.

No es un secreto para nadie que el pueblo indígena Misak es excluido territorialmente y geográficamente por influencias políticas $y$ el juego es una herramienta para fortalecer toda su sabiduría y saberes ancestrales, es la manera que ellos encuentran para mantener en equilibrio una cultura y unas costumbres que han sido afectadas por la violencia, fortalecer los saberes ancestrales logra en cada niño o niña despertar con el tiempo lo que quedó dormido en las familias que han sido permeadas por la cultura occidental.

\section{REFERENCIAS BIBLIOGRÁFICAS}

\author{
Allan, N., Wachholtz, D., \& Valdés, A. (2005). \\ Cambios en la Ocupación de los \\ adultos mayores recientemente \\ jubilados. Revista Chilena de terapia \\ ocupacional, (5), pág-19.
}

Braude, F. (2019). Hacia una pedagogía del disfrute. Revista El Anzuelo.

Cabildo, D. G. (2008). Segundo plan de vida de pervivencia y crecimiento Misak Mananasrekurri mananasrӨnkatik Misak waramik.

Cerchiaro-Ceballos, E., Barras-Rodríguez, R., \& Vargas-Romero, H. (2019). Juegos cooperativos y razonamiento prosocial en niños: efectos de un programa de intervención. Duazary, 16(3), 40-53.

Chiappe, S. M. D., Cardinal, M. C. M., González, J. M. P., \& Velásquez, E. L. C. (2019). Retratos del juego en Colombia: una mirada desde la documentación pedagógica. Hojas y Hablas, (17), 118135.

Coldeportes (2018). Lineamientos de política pública dirigidos a entes deportivos departamentales para el fomento de juegos tradicionales, autóctonos $y$ populares, actividades deportivas $y$ deporte convencional. Bogotá.

Corrales, M.H. (2018). Lenguas ancestrales de Colombia. Entre acallamientos y sobrevivencias, educaciones y escuelas. Ikastorratza. e-Revista de Didáctica, 20, 1- 32. Retrieved from http://www.ehu.es/ ikastorratza/20_alea/1.pdf

Del Toro Alonso V. (2012). El Juego en alumnos con necesidades educativas especiales: Síndrome de West yotras Encefalopatías Epilépticas. Tesis Doctoral. Universidad Complutense de Madrid. facultad de Educación. Departamento de Didáctica y Organización Escolar. Madrid.

Huizinga, J. (1972). Homo ludens. Madrid: Alianza Editorial, S.A. 
Husserl, E. (1997). Ideas relativas a una fenomenología pura y una filosofía fenomenológica. Libro primero. Introducción general a la fenomenología pura.

Lacasa, P. (2011). Los videojuegos. Ediciones Morata.

Loyola, L., \& María, A. (2016). El Juego Cooperativo y Habilidades sociales en estudiantes de 5 años, Barranca-2014.

Machuca, D. (2017). El conflicto armado a la luz de las incursiones guerrilleras. Análisis Político, 30(90), 229-231.

Medina, M. A. G., \& Cantero, C. L. (2018). Interculturalidad de las Etnias en Colombia.

Méndez-Giménez, A., \& Pallasá-Manteca, M. (2018). Disfrute y motivación en un programa de recreos activos. Apunts. Educación física y deportes, 4(134), 5568.

Meneses, M., \& Monge, M. D. (2001). El juego en los niños: enfoque teórico. Revista educación, 25(2), 113-124.

Muñoz \& Yalanda (2017) Los juegos propios que refuerzanlaidentidadMisak. Universidad Javeriana, facultad de humanidades y ciencias sociales, maestría en derechos humanos y cultura de paz. Trabajo de grado recuperado de http:// vitela.javerianacali.edu.co/bitstream/ handle/11522/10568/Juegos propios practicas.pdf?sequence $=1$ \&isAllowed $=y$

Padrón, J. (1998). La estructura de los procesos de investigación. Revista educación y ciencias humanas, 9(17), 33-45.

Panqueba-Cifuentes, J. F. (2012). Chaaj (Juego de pelota mesoamericano): Un juego ancestral entre emergencias culturales. Ra Ximhai, 8(3), 49-64.

Pugmire-Stoy, M. C. (1992). Spontaneous play in early childhood. Delmar Publishers.

Quispillo, L., Marinela, G., Cahuantico, B., \& Marisol, Y. (2020). Influencia del juego dramatico en las habilidades sociales de estudiantes de cuatro años de la IE Jaime White, Puerto Maldonado 2018 (Doctoral dissertation, Universidad Nacional Amazónica de Madre de Dios).

Ritzer, G. (1996). Sociological Beginnings: On the Origins of Key Ideas in Sociology. University of Maryland.

Salazar, R. D. (2018). Programa de actividades lúdicas para desarrollar habilidades sociales. Hacedor-AIAPAEC, 2(1), 7787.

Sampieri, R. H., Collado, C. F., Lucio, P. B., Valencia, S. M., \& Torres, C. P. M. (1998). Metodología de la investigación (Vol. 6). México, DF: Mcgraw-hill.

Shotter, J. (2001). Realidades conversacionales: la construcción de la vida a través del lenguaje.

Toledo, (2017). El valor del cuerpo para las culturas deportivas de los pueblos indígenas

Yuni, J., \& Urbano, C. (2005). Mapas y herramientas para conocer la escuela: Investigación etnográfica. Investigación Acción. 3era edición. Argentina: Barajas. 